\title{
Radial Scar/Complex Sclerosing Lesion
}

National Cancer Institute

\section{Source}

National Cancer Institute. Radial Scar/Complex Sclerosing Lesion. NCI Thesaurus. Code C9495.

A benign breast lesion characterized by the presence of fibrosis with stellate appearance in the parenchyma. The lesions are often multiple and bilateral. Grossly, the lesions are irregular and firm and resemble carcinoma. Microscopically, the stellate lesions are composed of dense, hyalinized collagen. Entrapped in the collagen are tubules. There is no evidence of cytologic atypia. 